\title{
PRESSURE DEPENDENCE OF RAMAN MODES IN THE CHALCOPYRITE QUATERNARY ALLOY $\mathrm{Ag}_{\mathrm{x}} \mathrm{Cu}_{1-\mathrm{x}} \mathrm{GaS}_{2}$.
}

\author{
In-Hwan Choi \\ Department of Physics, Chung-Ang University, Seoul, Korea \\ and \\ Peter Y. Yu ${ }^{(a)}$ \\ Department of Physics, University of California, Berkeley, \\ and \\ Materials Sciences Division, Lawrence Berkeley National Laboratory, \\ Berkeley, CA 94720.
}

\begin{abstract}
Raman scattering in the chalcopyrite quaternary alloy $\mathrm{Ag}_{\mathrm{x}} \mathrm{Cu}_{1-\mathrm{x}} \mathrm{GaS}_{2}$ has been studied under high pressure (up to $7 \mathrm{GPa}$ ) and at low temperature $(50 \mathrm{~K})$ using a diamond anvil high pressure cell for alloy concentrations $\mathrm{x}=1,0.75,0.5,0.25$ and 0 . This has allowed us to determine the dependence of their zone-center phonon modes on both pressure and alloy concentration. The resultant phonon pressure coefficients are helpful in understanding the nature of the phonon modes in these chalcopyrites.
\end{abstract}

PACS:78.55.Hx; 78.40.-q; 78.20.Hp

Substance Classification: S8.12

(a) email: PYYU@LBL.GOV; fax: 510-643-8497. 
The vibrational modes of semiconductors with the chalcopyrite structure and formula IIII- $\mathrm{VI}_{2}$, such as $\mathrm{CuGaS}_{2}$ and $\mathrm{AgGaS}$, have been investigated extensively by both Raman scattering and infrared absorption[1-5]. In particular, the pressure dependence of the zone-center optical phonon modes in $\mathrm{CuGaS}_{2}$ and $\mathrm{AgGaS}_{2}$ at room temperature have been reported[3,6]. More recently, alloys of these semiconductors have attracted attention as important materials for solar cells $[7,8]$. The alloy system $\mathrm{Ag}_{\mathrm{x}} \mathrm{Cu}_{1-\mathrm{x}} \mathrm{GaS}_{2}$ is interesting because its band gap exhibits a rather large bowing parameter and can be tuned over a range of $350 \mathrm{meV}$ by varying the amount of Ag. The optical and vibrational properties of these alloys have recently been studied by us as a function of alloy concentration[5]. In this paper we report a study of the effect of high pressure on the Raman modes in these alloys. From the pressure coefficients of the Raman modes we have obtained insights into the nature of the zone-center optical modes in these compounds. In $\mathrm{AgGaS}_{2}$ we have observed the splitting of some phonon peaks and the appearance of new peaks above 4.4 $\mathrm{GPa}$ as a result of a structural phase transition. This phase transition pressure is shifted to higher values in the alloys. Unlike the band gap the pressure coefficients of the phonon modes are found to have relatively small dependence on alloy concentration.

Our $\mathrm{Ag}_{\mathrm{x}} \mathrm{Cu}_{1-\mathrm{x}} \mathrm{GaS}_{2}$ (to be abbreviated as ACGS) samples, where $\mathrm{x}$ varies between 0 and 1 in steps of 0.25 , were grown with the horizontal Bridgman technique. The details of the sample growth and preparation processes have been presented elsewhere and therefore will not be repeated here[5]. High pressure optical measurements were performed inside a gasketed diamond anvil cell (DAC) using a mixture of methanol/ethanol as the pressure medium. The pressure was determined by the standard ruby fluorescence technique. The DAC was cooled to $50 \mathrm{~K}$ with a closed-cycle refrigerator. The Raman measurements were performed in a backscattering geometry 
using the 514.5 and $488.8 \mathrm{~nm}$ lines of an Ar ion laser. The unpolarized Raman signal was analyzed with a double Raman spectrometer equipped with a photon counting system.

The pressure dependence of the room temperature Raman modes in both $\mathrm{AgGaS}_{2}$ and $\mathrm{CuGaS}_{2}$ were reported by Carlone et al. [3]. Our low temperature results are, in general, in good agreement with their results. Figure 1 summarizes the pressure dependence of the Raman modes in $\mathrm{AgGaS}_{2}$. The identifications of the observed Raman modes in Fig. 1 are based on those proposed by Carlone et al.[3]. The existence of a structural phase transition around $4.4 \mathrm{GPa}$ is evident from a splitting of the mode labeled as $\mathrm{D}$ and the appearance of a new peak $\mathrm{C}$. The relation between these Raman features and the structural phase transition is still unknown. A similar structural phase transition was reported by Carlone et al. [3] in $\mathrm{CuGaS}_{2}$ at the higher pressure of 16.5 GPa. This transition is beyond the lower pressure range of the present experiment.

We found evidence of a phase transition also in the alloy $\mathrm{Ag}_{0.5} \mathrm{Cu}_{0.5} \mathrm{GaS}_{2}$. The pressure dependent Raman spectra in this sample are shown in Fig. 2. The Raman frequencies of the observed modes are plotted as a function of pressure in Fig. 3. We note first that most of the Raman modes in the ACGS alloys are strongly broadened[5]. The exception is the totally symmetric $\Gamma_{1}$ peak which can be observed throughout the entire range of alloy concentrations. In the alloy $\mathrm{Ag}_{0.5} \mathrm{Cu}_{0.5} \mathrm{GaS}_{2}$ we also found strong variations in the Raman intensity as result of resonance effects. The band gap of $\mathrm{Ag}_{0.5} \mathrm{Cu}_{0.5} \mathrm{GaS}_{2}$ at low temperature is around $2.45 \mathrm{eV}$. A pressure of around $2.2 \mathrm{GPa}$ will increase its energy gap to $\sim 2.53 \mathrm{eV}$ which is almost in resonance with the $488.8 \mathrm{~nm}$ laser line[9]. This explains why three broad features in the alloy Raman spectrum in Fig. 2 become enhanced enough at $2.2 \mathrm{GPa}$ to become comparable in intensity to the $\Gamma_{1}$ peak. A structure with this typical behavior is the one we have labeled A in Fig. 2 and centered 
below the $\Gamma_{1}$ peak at a frequency of $\sim 260 \mathrm{~cm}^{-1}$. These peaks become weaker at higher pressures until they become unobservable at 7.3 GPa. A peak (labeled B in Fig. 2) with a completely different pressure dependence appears in the $6.0 \mathrm{GPa}$ spectrum. The strength of this peak grows with pressure and becomes almost as strong as the dominant $\Gamma_{1}$ peak at $7.3 \mathrm{GPa}$. The pressure dependence of this peak $B$ is rather similar to that of the peak $C$ in Fig. 1. If we take the appearance of the peak B as evidence of a pressure-induced phase transition, as in $\mathrm{AgGaS}_{2}$, then the transition pressure in the alloy $\mathrm{Ag}_{0.5} \mathrm{Cu}_{0.5} \mathrm{GaS}_{2}$ is estimated to be $\sim 6 \mathrm{GPa}$. No similar appearance of pressure-induced peaks are observed in the other two alloys. One possibe reason is that such peaks are too weak to be observed without the resonance enhancement in $\mathrm{Ag}_{0.5} \mathrm{Cu}_{0.5} \mathrm{GaS}_{2}$.

For simplicity, we have fitted the measured Raman frequencies $\omega$ in Figs. 1 and 3 as linear functions of pressure: $\omega=\omega_{0}+\alpha \mathrm{P}$ (where $\alpha$ is the linear pressure coefficient) although some of the modes, such as the peak D in Fig. 1, exhibit a small nonlinear dependence on pressure at higher pressures. The resultant pressure coefficients are listed in Figs. 1 and 3 for $\mathrm{AgGaS}_{2}$ and the alloy $\mathrm{Ag}_{0.5} \mathrm{Cu}_{0.5} \mathrm{GaS}_{2}$. For $\mathrm{AgGaS}_{2}$ (and also for $\mathrm{CuGaS}_{2}$ although the results will not be shown here) one finds that the Raman modes can be divided roughly into three groups according to the magnitude of their $\alpha$. The first group includes the low frequency modes with $\omega_{0}<200 \mathrm{~cm}^{-1}$. Their $\alpha$ are either negative or $\sim 1 \mathrm{~cm}^{-1} / \mathrm{GPa}$. The small or negative $\alpha$ can be understood by the fact these modes correspond to the zone-edge acoustic modes in the zincblende-structure semiconductor "folded" into zone-center in the chalcopyrite structure[10]. The zone-edge acoustic modes in the zincblende semiconductors are well-known to have negative $\alpha$ because of the instability of the zincblende lattice[11]. Since these modes in the chalcopyrites involve motion 
of the heavier elements, they are heavily damped by alloying $\mathrm{Ag}$ with $\mathrm{Cu}$. The next group of modes includes the intermediate frequency phonons with an intermediate value of $\alpha \sim 4-5$ $\mathrm{cm}^{-1} / \mathrm{GPa}$. These modes in the chalcopyrite structure can be thought of arising from "folded" zone-edge optical phonons in the zincblende structure. Their values of $\alpha$ are smaller than the zone-center phonons because they still contain some admixture of the "folded" zone-edge acoustic modes. The admixture tends to be larger for the lower frequency modes and hence the value of $\alpha$ increases with the mode frequency. Finally, the values of $\alpha$ are of the order of 6 $\mathrm{cm}^{-1} / \mathrm{GPa}$ for the highest frequency modes. These modes involve mostly motion of the lightest element, namely, $\mathrm{S}$ in the cyrstal lattice and as a result their frequencies are not sensitive to alloying[10]. These high frequency modes are observable in all the alloys[5] inspite of significant broadening by alloy fluctuation. This picture is supported by the pressure dependence of the high frequency modes in the alloy $\mathrm{Ag}_{0.5} \mathrm{Cu}_{0.5} \mathrm{GaS}_{2}$. The values of $\alpha$ for the two high frequency modes in Fig. 3 are $~ 5.5 \mathrm{~cm}^{-1} / \mathrm{GPa}$.

It is worthwhile noting that the only Raman peak which remains unbroadened by alloying is the totally symmetric $\Gamma_{1}$ mode. Both its frequency and $\alpha$ increase monotonically from $\mathrm{AgGaS}_{2}$ to $\mathrm{CuGaS}_{2}$. The alloy dependence of this mode can be truly classified as a "one-mode" behavior [12] as in the zincblende-type alloy InGaAs.

In conclusion, we have studied the pressure dependence of the Raman modes in the chalcopyrite alloys of $\mathrm{Ag}_{\mathrm{x}} \mathrm{Cu}_{1-\mathrm{x}} \mathrm{GaS}_{2}$. We have found evidence of a phase transition in the alloy $\mathrm{Ag}_{0.5} \mathrm{Cu}_{0.5} \mathrm{GaS}_{2}$. The pressure dependence of the Raman modes in the alloys supports the proposal that the highest frequency modes in both $\mathrm{AgGaS}_{2}$ and $\mathrm{CuGaS}_{2}$ involve mostly motion of the sulfur atoms and, therefore, are not sensitive to alloying of the group I cations. 


\section{Acknowledgments}

The part of this work performed in Korea was supported by the Chung-Ang University Special Research Grants in 1998. The work at Berkeley was supported by the Director, Office of Energy Research, Office of Basic Energy Sciences, Materials Sciences Division, of the US Department of Energy under Contract No. DE-AC03-76SF00098. IHC also acknowledges a fellowship from the Korea Ministry of Education through Research Fund BSRI-96-2453. 


\section{REFERENCES}

[1] J. P. van der Ziel, A. E. Meixner, H. M. Kasper, and J. A. Ditzenberger, Phys. Rev. B 9, 4286 (1974).

[2] W. H. Koschel and M. Bettini, Phys. Status Solidi b 72, 729 (1975).

[3] C. Carlone, D. Olego, A. Jayaraman and M. Cardona, Phys. Rev. B 22, 3877 (1980).

[4] S. Sugai, J. Phys. Soc. Jpn. 43, 5992 (1977).

[5] I-H. Choi, S-H. Eom and P. Y. Yu, J. Appl. Phys. 87, 3815 (2000).

[6] M. Bettini and W. B. Holzpafel, Solid State Commun. 16, 27 (1975).

[7] J. R. Sites, and X-X. Liu, Solar Energy Materials \& Solar Cells, 41/42, 373 (1996).

[8] I-H. Choi, and P. Y. Yu, Phys. Rev. B55, 9642 (1997).

[9] See another paper by I-H. Choi and P. Y. Yu in this conference.

[10] L. Artus, J. Pujol, J. Pascual, and J. Camassel, Phys. Rev. B 41, 5727 (1990).

[11] See, for example, Peter Y. Yu and B. Welber, Solid State Commun. 25, 209 (1978).

[12] See, for example, L. Genzel, T. P. Martin and C. H. Perry, Phys. Stat. Solidi b 62, 83 (1974). 


\section{FIGURE \& CAPTIONS}

Figure 1 The low temperature Raman frequencies of optical phonons in $\mathrm{AgGaS}_{2}$ as a function of pressure.

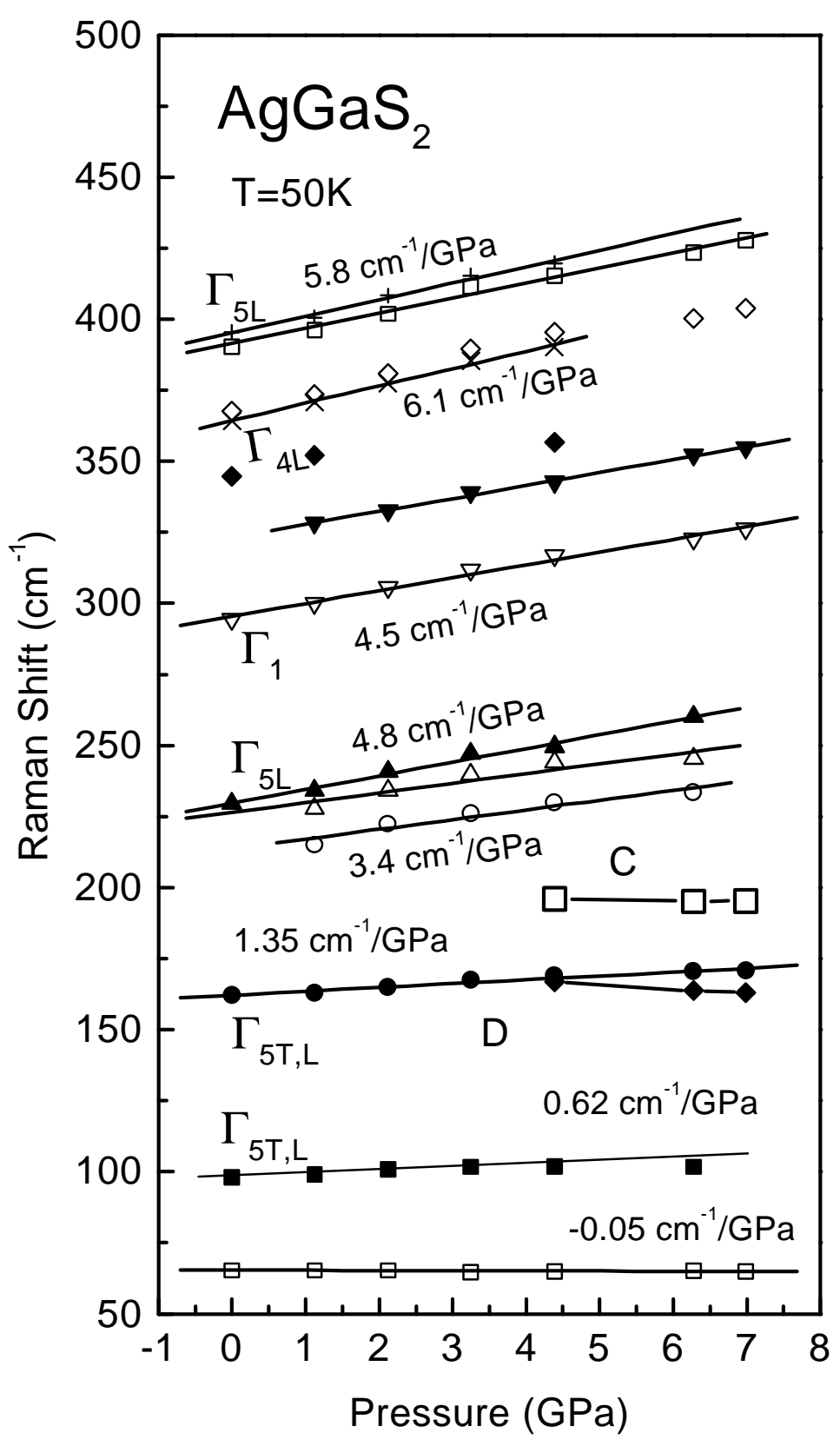


Figure 2 The low temperature Raman spectra of $\mathrm{Ag}_{0.5} \mathrm{Cu}_{0.5} \mathrm{GaS}_{2}$ for several different pressures. The arrows indicate the energy of the two peaks A and B which showed strong dependence on pressure.

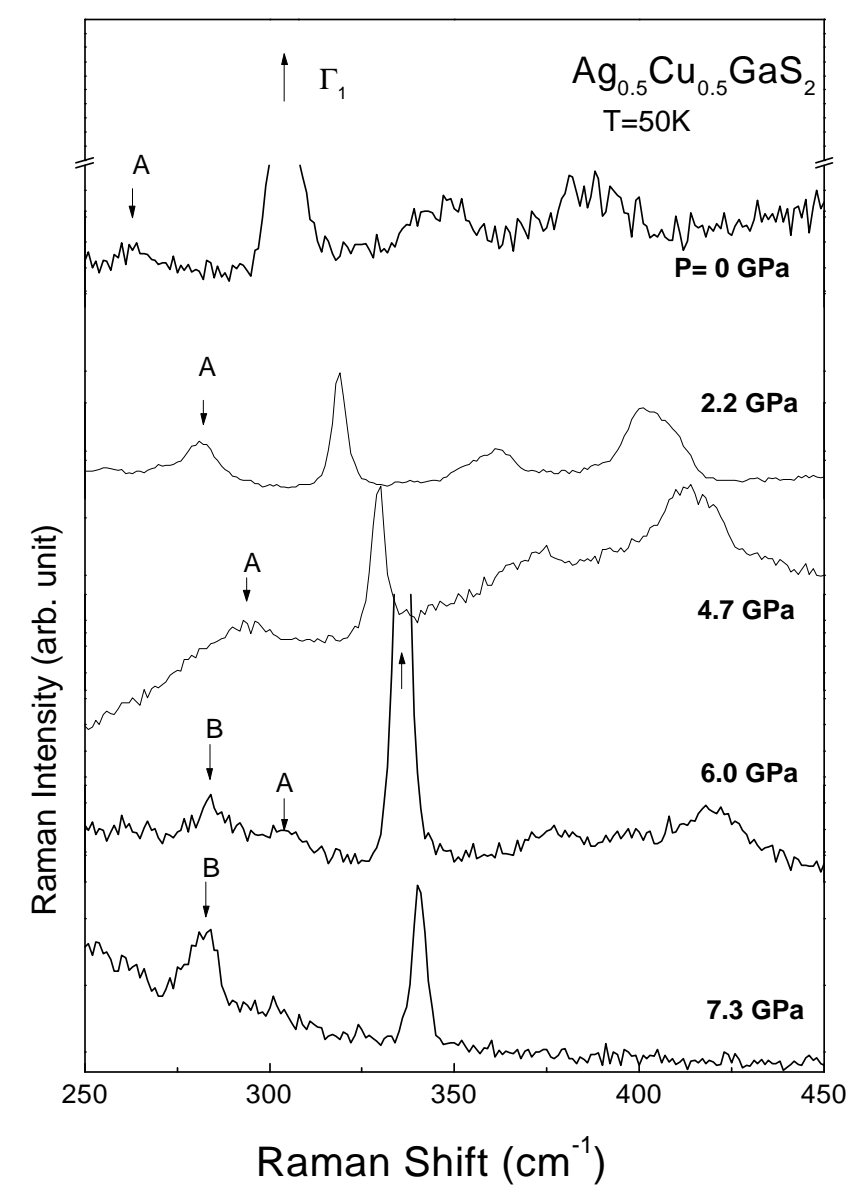


Figure 3 The pressure dependence of the frequencies of Raman modes in $\mathrm{Ag}_{0.5} \mathrm{Cu}_{0.5} \mathrm{GaS}_{2}$.

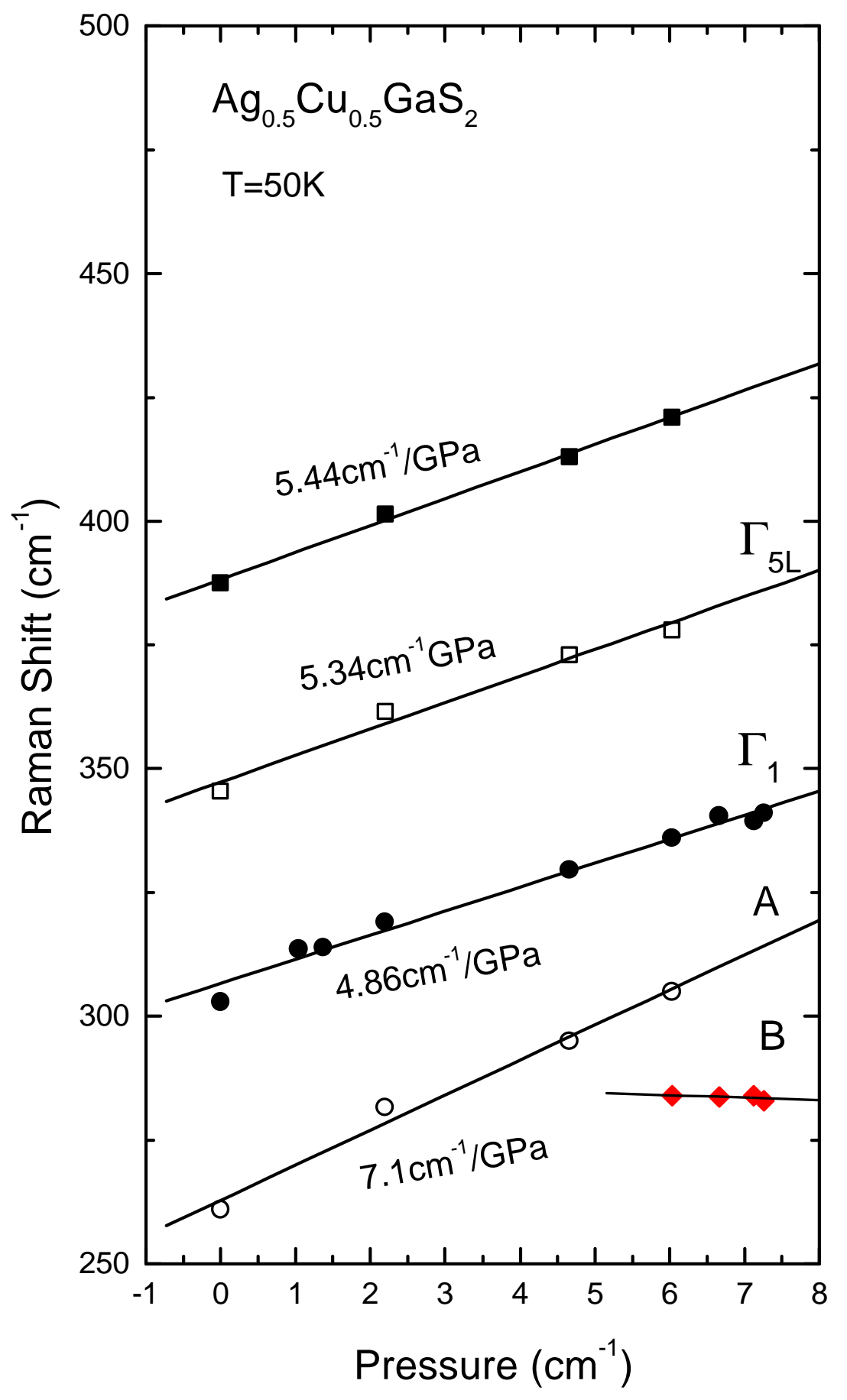

